\title{
Revisão da rota das contribuições nacionalmente determinada no peru para as mudanças climáticas
}
A review of the route of the national determined contributions in peru to climate change

Revisión a la ruta de las contribuciones nacionalmente determinadas en el perú al cambio climático

María Teresa Alejandra Diaz Figueroa

Engenheira Ambiental e Mestranda do PROAC, UFJF, NAGEA, Brasil diaz.alejandra@engenharia.ufjf.br

\section{Cézar Henrique Barra Rocha} Professor Titular da UFJF, Departamento de Transportes e Geotécnica, NAGEA, PPGEO, PROAC, Brasil. barra.rocha@engenharia.ufjf.br

Hilda Evita Mendoza Soto Engenheira Ambiental, UNALM, PROFONAMPE, Peru. mendozasoto.hildaevita@gmail.com 


\section{RESUMO}

O Peru é um país vulnerável às mudanças climáticas e às condição vem se agravando devido aos impactos ambientais negativos de origem antrópica que causam as mudanças climáticas. Além disso, a pandemia da COVID-19 marcou impactos em todo o mundo e no Peru, que desenvolvem eventos desastrosos para o meio ambiente. Como consequência do diálogo internacional e da preocupação dos países integrantes da Convenção-Quadro das Nações Unidas sobre Mudança do Clima em enfrentar esse problema, foi assinado o Acordo de Paris. 0 objetivo principal deste artigo é desenvolver uma revisão das ações mais importantes que enquadram as Contribuições Nacionalmente Determinadas do Peru, destacando-se os impactos no contexto da pandemia da COVID-19 e conhecendo os impactos da COVID-19 na Agenda 2030 e seus 17 Objetivos de Desenvolvimento. Para tanto, foi realizada uma revisão bibliográfica dos documentos realizados pelas diversas instituições envolvidas, procurando compreender sob diversos pontos de vista, o desenvolvimento das ações para cumprir os compromissos assumidos frente às mudanças climáticas, assim como evidenciar as diversas medidas políticas e técnicas adotadas para o cumprimento dos objetivos assumidos no referido Acordo. Da mesma forma, foram evidenciados alguns elementos que não agregam esforços para cumprir essas contribuições e outros elementos a serem analisados. Este documento pode contribuir para uma compreensão sucinta da rota das Contribuições Nacionalmente Determinadas do Peru.

PALAVRAS-CHAVE: Contribuições Nacionalmente Determinadas, Mudanças Climáticas, Pandemia da COVID-19.

\section{ABSTRACT}

Peru is a vulnerable country in the face of climate change and its condition has been worsening due to the negative environmental impacts of anthropic origin that cause climatic changes. In addition, the COVID-19 pandemic marked impacts around the world and in Peru, which develop disastrous events for the environment. As a consequence of the international dialogue and the concern for the countries belonging to the United Nations Framework Convention on Climate Change, in order to face this problem, the Paris Agreement was signed. The main objective of this article is to develop a review of the most important actions that frame Peru's Nationally Determined Contributions, highlighting the impacts in the context of the COVID-19 pandemic and knowing the impacts of COVID-19 on the 2030 Agenda and its 17 Objectives of development. Therefore, a bibliographic search was carried out in the documents prepared by various institutions involved, seeking to understand from different points of view, the development of actions to comply with the commitments assumed in the face of climate change and to show the different political and technical measures adopted to fulfill the goal assumed in the aforementioned Agreement. Likewise, some elements that do not add to the efforts to fulfill these contributions and other elements to analyze were evidenced. This document can help to understand in a succinct way, the route of the Nationally Determined Contributions of Peru.

KEY WORDS: Nationally Determined Contributions, Climate Change, COVID-19 pandemic.

\section{RESUMEN}

Perú es un país vulnerable frente al cambio climático y su condición se viene agravando debido a los impactos ambientales negativos de origen antrópico que ocasionan alteraciones climáticas. Además, la pandemia de COVID-19 marcó impactos en todo el mundo y en el Perú, que ocasionaron eventos desastrosos para el medio ambiente. Como consecuencia del diálogo internacional y la preocupación por los países pertenecientes a la Convención Marco de las Naciones Unidas sobre el Cambio Climático, por encarar este problema, se firmó el Acuerdo de París. El objetivo principal de este artículo es desarrollar una revisión de las acciones más importantes que enmarcan las Contribuciones Determinadas a Nivel Nacional del Perú, destacando los impactos en el contexto de la pandemia COVID-19 y conociendo los impactos del COVID-19 en la Agenda 2030 y sus 17 Objetivos de desarrollo. Por tanto, se llevó a cabo una búsqueda bibliográfica en los documentos elaborados por diversas instituciones involucradas, buscando comprender de distintos puntos de vista, el desenvolvimiento de acciones para cumplir los compromisos asumidos frente al cambio climático y evidenciar las diferentes medidas políticas y técnicas adoptadas para cumplir la meta asumida en el mencionado Acuerdo. Asimismo, se evidenciaron algunos elementos que no suman a los esfuerzos por cumplir estas contribuciones y otros elementos para analizar. Este documento puede contribuir a comprender de manera sucinta, la ruta de las Contribuciones Nacionalmente Determinadas del Perú.

PALABRAS CLAVE: Contribuciones Nacionalmente Determinadas, Cambio Climático, Pandemia del COVID-19. 


\title{
Revista Científica ANAP Brasil
}

\author{
ISSN 1984-3240 - Volume 14, número 32, 2021
}

\section{INTRODUÇÃO}

O Peru é um país "particularmente vulnerável" aos impactos negativos das mudanças climáticas, pois inclui sete características identificadas pela Convenção-Quadro das Nações Unidas sobre Mudanças Climáticas (UNFCCC): áreas costeiras baixas; zonas áridas e semi-áridas; áreas expostas a secas e desertificação; áreas sujeitas a desastres naturais; áreas com alta poluição do ar urbano e economias que dependem fortemente da renda gerada pela obtenção e uso de combustíveis fósseis; e frágeis ecossistemas de montanha.

A situação climática do país vem se agravando devido aos impactos ambientais antrópicos negativos, existem estudos que demostram que as temperaturas e as chuvas estão mudando em todo o território peruano, prevendo irregularidades nos regimes hídricos até 2030. Cerca de $24 \%$ da população peruana é rural, sendo que as áreas rurais e ocupadas por povos indígenas têm um desenvolvimento baseado na produção primária e extrativista, podendo comprometer ecossistemas vulneráveis. Aproximadamente $80 \%$ da População Economicamente Ativa (PEA) rural vive na pobreza e a principal atividade econômica é a agricultura, mineração e pesca. Somam-se a essa situação, eventos extremos não previstos como o fenômeno El Niño do ano 2017, ou os incêndios florestais na Amazônia, que alteram o ciclo do carbono e, embora o desmatamento tenha diminuído nos últimos anos, os incêndios são mais frequentes.

Além disso, a pandemia da COVID-19 marcou impactos em todo o mundo e no Peru, que desenvolvem eventos desastrosos para o meio ambiente, tais como: aumento do desmatamento para fins agrícolas (plantações ilegais de coca, Cannabis e papoila) e incremento da mineração ilegal; extração ilegal de madeira; tráfico de espécies em risco de extinção; invasão de áreas naturais protegidas e terras de comunidades indígenas; geração não controlado de resíduos sólidos de todos os tipos; e sobre-exploração dos recursos marinhos e fluviais (Dourojeanni, 2020). Esses problemas ambientais se agravaram durante a pande mia, que terá consequências de curto, médio e longo prazo que minimizam a pequena desaceleração no aumento das emissões de gases de efeito estufa ( LIU et al., 2020).

No âmbito das Contribuições Nacionalmente Determinadas (NDC), os países com realidades diferentes, desenvolvidos, emergentes, em desenvolvimento, com economias de extrema pobreza e de maior vulnerabilidade se preparam para enfrentar as mudanças climáticas, alinhando-se com o objetivo de se reorientar para a sustentabilidade, resiliência, economia verde, descarbonização, preocupação e cuidado com os mais vulneráveis.

\section{OBJETIVO}

O objetivo geral deste trabalho é revisar a ações mais importantes que enquadram as Contribuições Nacionalmente Determinadas do Peru, com ênfase no contexto da pandemia da COVID-19.

Os objetivos específicos deste trabalho são:

a) Identificar as ações realizadas pelo Estado peruano para alcançar os objetivos frente às mudanças climáticas.

b) Conhecer os impactos originados pela pandemia da COVID-19 no contexto da mudança climática. 
c) Conhecer os impactos da COVID-19 na Agenda 2030 e seus 17 Objetivos de Desenvolvimento Sustentável(ODS).

\section{MÉTODO DE ANALISE}

O presente trabalho foi desenvolvido com a metodologia da pesquisa básica, que segundo Schwartzman (1979) define como "aquela que acumula conhecimentos e informações que podem eventualmente levar a resultados acadêmicos ou aplicados importantes, mas sem fazê-lo diretamente".

Por tanto, é realizada uma revisão bibliográfica nos documentos realizados pelas diversas instituições envolvidas, procurando compreender a partir de diferentes pontos de vista as diferentes medidas políticas e técnicas adotadas para cumprir o objetivo assumido no Acordo de Paris.

\section{RESULTADOS E DISCUSSÕES}

\subsection{O caminho para o Acordo de Paris}

O Peru e a França atuaram de forma colaborativa para promover a 21ํㅡ Conferência das Partes (COP21) realizada em Paris em 2015. Essa conferencia reuniu representantes de 195 países para a concretização do Acordo de Paris cujo objetivo era melhorar e substituir o conhecido Protocolo de Quioto assinado na COP3 em 1997; esse novo Acordo climático associado e global estabelece oficialmente a meta de: "manter o aumento da temperatura média global abaixo de 2 graus Celsius em relação aos níveis pré-industriais, além de continuar os esforços para limitar esse aumento de temperatura a 1,5 graus Celsius em relação aos níveis pré-industriais".

O Acordo de Paris afirma que deve existir uma maior disposição das partes para se adaptar aos resultados adversos das mudanças climáticas, promovendo a redução das emissões de carbono. Do mesmo modo admite que garantirá que os fundos sejam destinados a ajudar os países em desenvolvimento, particularmente vulneráveis às consequências das mudanças climáticas. Uma das disposições deste acordo são as NDC, que consistem em medidas internas e específicas que cada país deve adotar e comunicar, conforme indicado no artigo 4음 do Acordo de Paris. Com este compromisso assumido, o Peru deve abordar ações de adaptação e mitigação das mudanças climáticas no território peruano, cujas realizações serão anunciadas periodicamente (Trinidad e Ortiz, 2019).

\subsection{Resposta do Peru à mudança climática}

De acordo com o último relatório do Painel Intergovernamental sobre Mudanças Climáticas (IPCC), adaptação refere-se ao desenvolvimento do ajuste climático projetado e as consequências de forma a minimizar seus danos e alcançar oportunida des positivas para o país, com particularidades em relação ao lugar e ao contexto. Enquanto, a mitigação basicamente enquadra a redução das emissões de gases de efeito estufa (GEE). 
Essas medidas visam promover a capacidade de competir com o auxílio de três tipos de medidas em diferentes setores da economia: (a) Medidas que geram economia ou eficiência no uso de recursos; (b) Medidas que evitem perdas ou maximizem o investimento do Estado e valorizem os recursos naturais; e (c) Medidas que atendem os novos nichos de mercado e se alinhem às tendências globais em um mundo com restrição e impacto de carbono, o que leva a mercados ou consumidores mais rigorosos, gerenciando novos desafios. Além disso, visa melhorar o comportamento socioambiental do país que deve se responsabilizar por sua pegada de emissões e pelo uso de recursos que impactam o clima e suas reservas (MINAM, 2015).

As NDC peruanas foram atualizadas no final de 2020, refletindo uma maior ambição climática e tornando-se metas absolutas. Esta atualização considera a redução de $40 \%$ das emissões de GEE calculadas para o ano de 2030 , sendo que $10 \%$ iriam depender da disponibilidade de financiamento externo e $30 \%$ dos financiamentos e desembolsos com recursos internos, privados e públicos do país (República do Peru, 2020). No ano 2015, foi apresentada a meta de emissões de GEE por uma quantidade de 298, 3 milhões de toneladas de dióxido de carbono equivalente $\left(\mathrm{CO}_{2}\right)$. No entanto, nesta atualização, o Estado peruano se compromete a que suas emissões líquidas de GEE não ultrapassem 208,8 milhões de toneladas de dióxido de carbono equivalente $\left(\mathrm{CO}_{2}\right)$ sem considerar financiamento externo e 179,0 milhões de toneladas de dióxido de carbono equivalente $\left(\mathrm{CO}_{2}\right)$ considerando o financiamento externo internacional.

Desde o seu início, foram realizados processos como a Consulta Pública Nacional, realizando reuniões informativas e oficinas regionais, em um processo denominado "Dialoguemos". Da mesma forma, foram elaborados estudos técnicos que permitiram fornecer informações às equipes técnicas de cada setor e às autoridades (Pulgar, 2016). Nesse sentido, pode-se afirmar que o Peru possui um esquema participativo, político e técnico na ge stão das mudanças climáticas. No ano 2017, foi criado um Grupo de Trabalho Multissetorial temporário (GTM-NDC), com a função de produzir informações técnicas para direcionar a implantação da NDC, constituído pelos treze Ministérios do Estado Peruano. A função deste grupo de trabalho é a articulação e compromisso entre os diferentes setores do governo.

O Peru propôs uma grande variedade de estratégias, políticas e planos para as NDC, apostando na melhor gestão dos recursos e não em ações que requeiram reparos tecnológicos. Esta é uma decisão economicamente razoável já que tais tecnologias são de alto custo e agravam o problema da demanda excessiva de energia e o risco de catástrofes. O componente de risco das NDC é que a maior parte dos investimentos necessários é privado, mesmo que as medidas sejam incentivadas e regulamentadas pelo estado (Raez, 2019).

\subsection{Ações em destaque}

Uma iniciativa importante no âmbito das mudanças climáticas é o Programa Nacional de Conservação de Florestas, instituído pelo Ministério do Meio Ambiente do Peru (MINAM) no ano 2010, por meio do Decreto Supremo № 008-2010-MINAM, cujo objetivo é conservar 54 milhões de hectares de florestas tropicais como contribuição para mitigar as mudanças climáticas e promover o desenvolvimento sustentável. As ações baseiam-se no fortalecimento das capacidades de conservação florestal e minimização do desmatamento nas comunidades nativas; fornecer informações oficiais e precisas sobre as mudanças na cobertura florestal; 
promover ações sustentáveis que ajudem a manter as florestas e melhorar as condições de vida das populações nativas; reforçar e coordenar a gestão entre atores públicos, privados e organizações indígenas; promover pesquisas acadêmicas e científicas para melhorar o conhecimento da floresta (MINAM, 2020).

O Inventário Nacional de Gases de Efeito Estufa (INGEI) também é uma das iniciativas de destaque, que fazem parte dos Relatórios Bienais de Atualização (BUR). O mais recente foi apresentado ao Secretariado das Nações Unidas em dezembro de 2019. A partir deste relatório correspondente ao ano de 2014, refletem que as emissões totais do país alcançam a 167.629,8 $\mathrm{Gg} \mathrm{CO}_{2} \mathrm{eq}$ (mil toneladas de $\mathrm{CO}_{2}$ ). Além de que foi determinado que 44,9\% (75.345,5 $\mathrm{GgCO}_{2} \mathrm{eq}$ ) correspondem ao setor de Uso da Terra, Mudança no uso da terra e silvicultura (USCUSS), cuja maior fonte de emissão são terras convertidas em terras agrícolas. Cerca do 30,0\% (50.331,2 $\mathrm{GgCO}_{2}$ eq) correspondem ao setor de Energia, cuja principal fonte de emissão é a queima de combustível para transporte. O 15,6\% para o setor Agropecuário (26.233,2 GgCO2eq), cuja principal fonte de emissão são os solos agrícolas. O 5,8\% para o setor de Resíduos $(9.679,7$ GgCO2eq), cuja principal fonte de emissão é a gestão de resíduos sólidos. E o 3,6\% para o setor de Processos Industriais e Uso do Produto - PIUP (6.040,8 GgCO2eq), cuja principal fonte de emissão é a indústria de minerais (MINAM, 2019).

O Governo do Peru criou a Lei-Quadro sobre Mudança do Clima (Lei no 3.0754) em abril de 2018 e posteriormente os regulamentos (DS no 013-2019-MINAM). Também foi criada a Comissão Multissetorial, durante a pandemia, de caráter permanente dependente do MINAM, denominada "Comissão de Alto Nível sobre Mudanças Climáticas", por meio do Decreto Supremo № 006-2020-MINAM, composta por Altos funcionários dos setores envolvidos. O objetivo desta Comissão é implementar medidas de adaptação e mitigação as mudanças climáticas contidas nas NDC. Da mesma forma, a Comissão Nacional de Mudanças Climáticas, a Plataforma dos Povos Indígenas e a plataforma digital Cadastro Nacional de Medidas de Mitigação (RENAMI) foram criadas para se juntar à ação climática.

O MINAM vem preparando um estudo técnico de Neutralidade do Carbono para o ano 2050 incluindo energias renováveis, eletrificação da economia, economia circular e soluções baseadas na natureza, como os principais pilares; com o objetivo de renovar a Estratégia Nacional para as Mudanças Climáticas em vigor até o ano 2021.

Da mesma forma, no setor de Energia na última década, impulsionado pelo constante aumento da demanda de energia e potencial energético latente no país, e não precisamentepor questões de mudanças climáticas, o Peru tem realizado investimentos significativos em tecnologias de energia solar, eólica, pequenas hidrelétricas e biomassa na América Latina (Vásquez e Gamio, 2018). Em abril de 2018, a Usina Solar Fotovoltaica Rubí entrou em operação com capacidade de 180 megawatts em corrente contínua (MWp), a maior do país, iniciou suas operações como resposta às mudanças climáticas. Existem também parques eólicos no norte do país, usinas hidrelétricas e projetos de bioenergia (MINAM, 2020).

As NDC peruanas dão maior importância à energia renovável e aos componentes de transporte sustentável. As primeiras conclusões obtidas das NDC estimou o potencial de minimização das emissões de GEE em medidas de mitigação relacionadas às energias renováveis e considera a inclusão de 6,8\% das usinas de geração de energia renovável na matriz elétrica. Porém, o objetivo do setor foi de aumentar até $15 \%$ a contribuição das energias renováveis. Nesse sentido, foi solicitada a elaboração de estudos que possibilitem superar as barreiras regulatórias ainda existentes, a fim de preservar a competitividade do custo da energia elétrica 


\section{Revista Científica ANAP Brasil}

ISSN 1984-3240 - Volume 14, número 32, 2021

no país. Da mesma forma, uma ótima infraestrutura elétrica, como as atuais linhas de transmissão, será fundamental para que possam manter a operação das novas usinas de energia renovável em função de sua descontinuidade. A melhoria das tecnologias permite uma maior utilização das energias renováve is ainda não competitivas no mercado, como as tecnologias de biomassa, o que não é o caso das tecnologias solares, pois tem se mostrado que nos últimos anos há uma tendência de redução de custos, alcançando uma redução de $70 \%$ desde o ano 2009 (S/. $18.760 / \mathrm{kW}$ ) até o 2016 (S/.5.610 /kW) (Gobierno del Perú, 2018).

A Comissão de Alto Nível sobre Mudanças Climáticas preparou um Estudo Técnico sobre as NDC para o período 2021-2030, parte de sua análise aborda as condições e desafios facilitadores da implementação de medidas de mitigação e adaptação. Em relação ao primeiro, identificaram 49 das principais condições de habilitação para todos os setores, que se encontram no seguinte estado: 37 (75,5\%) em implantação; 9 (18,4\%) pendentes; 3 (6,1\%) executados. Das 49 condições de habilitação: o setor de Energia tem 7 (63,6\%) em implantação, $2(18,2 \%)$ pendentes e $2(18,2 \%)$ executadas; o setor PIUP tem $8(88,9 \%)$ em implantação e 1 (11,1\%) pendente; o setor de Resíduos, 9 (64,3\%) em implantação, 4 (28,6\%) pendentes e 1 $(7,1 \%)$ executada; o setor USCUSS, 7 (77,8\%) em implantação e 2 (22,2\%) pendentes; e Agricultura, 6 em implantação (100\%) (Gobierno del Perú, 2020).

\subsection{Os impactos do COVID-19 nas ações de mudança climática}

Os impactos da COVID-19 têm efeitos na sociedade e no meio ambiente com mudanças no estilo de vida da população mundial em tempo recorde, ou seja, o mundo caminha para um futuro sustentável com maior resiliência nos sistemas socioecológicos. No entanto, os países envolvidos no Acordo de Paris também têm a opção de crescimento econômico, deixando de lado a sustentabilidade (Cheval et al., 2020). No quadro 1, podem ser observados os impactos negativos, neutros e positivos da COVID-19, sendo que os blocos vermelhos são impactos negativos, os verdes são positivos e os cinzas representam os efeitos neutros. 
Quadro 1- Matriz de impactos observados e potenciais da COVID-19 no meio ambiente e nas mudanças climáticas.

\begin{tabular}{|c|c|c|c|c|}
\hline & Días da semana & Meses & \multicolumn{2}{|l|}{ Anos } \\
\hline \multirow[t]{2}{*}{$\begin{array}{l}\overline{0} \\
\frac{0}{0} \\
\end{array}$} & \multirow{2}{*}{$\begin{array}{l}\text { Redução dos dados } \\
\text { observados e capacidade } \\
\text { de monitoramento. }\end{array}$} & $\begin{array}{l}\text { Mudanças sociais e } \\
\text { econômicas com o } \\
\text { impacto ambiental. }\end{array}$ & \multicolumn{2}{|c|}{ Políticas ambientais e de mudança climática. } \\
\hline & & \multicolumn{2}{|c|}{ Pesquisas ambientais para aplicações epidemiológica } & \multirow{8}{*}{$\begin{array}{c}\text { Melhoria do } \\
\text { planejamento } \\
\text { para a redução } \\
\text { do risco de } \\
\text { desastres }\end{array}$} \\
\hline \multirow{4}{*}{ 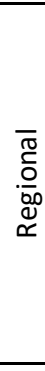 } & $\begin{array}{l}\text { Diminuição da poluição } \\
\text { ambiental devido a redução } \\
\text { do trânsito. }\end{array}$ & \multicolumn{2}{|c|}{$\begin{array}{l}\text { Melhoramento dos sistemas para monitoramento } \\
\text { ambiental }\end{array}$} & \\
\hline & $\begin{array}{c}\text { Poluição do litoral devido a } \\
\text { deposição sanitária. }\end{array}$ & $\begin{array}{c}\text { Política climática } \\
\text { regional }\end{array}$ & Melhoria do clima regional & \\
\hline & & $\begin{array}{c}\text { Planos de adaptação } \\
\text { aprimorados }\end{array}$ & $\begin{array}{l}\text { Melhoria do bem-estare } \\
\text { proteção da saúde da } \\
\text { população }\end{array}$ & \\
\hline & \multicolumn{2}{|c|}{ Restauração ecológica } & $\begin{array}{c}\text { Mudanças na participação } \\
\text { do consumo da água }\end{array}$ & \\
\hline \multirow{4}{*}{$\overline{\widetilde{J}}$} & $\begin{array}{c}\text { Diminuição da poluição } \\
\text { acústica. }\end{array}$ & & & \\
\hline & $\begin{array}{l}\text { Incremento da poluição da } \\
\text { água e do solo devido ao } \\
\text { aumento dos resíduos }\end{array}$ & \multicolumn{2}{|c|}{ Planos de adaptação aprimorada para cidades } & \\
\hline & \multicolumn{2}{|c|}{ Melhoria da qualidade do ar em áreas urbanas } & $\begin{array}{c}\text { Mudanças no } \\
\text { comportamento da } \\
\text { sociedade }\end{array}$ & \\
\hline & Imediato & Curto prazo & Longo praz & \\
\hline
\end{tabular}

Fonte: Adaptado do Cheval et al., 2020.

Os impactos potenciais e observados devido à pandemia da COVID-19 refletidos no Quadro 1 também podem ser entendidos como o impacto da COVID-19 na Agenda 2030 e seus 17 Objetivos de Desenvolvimento Sustentável (ODS).

\subsection{Os impactos do COVID-19 na Agenda 2030 e seus 17 Objetivos de Desenvolvimento Sustentável (ODS)}

Vários autores (Cheval et al., 2020) concordam que todos os esforços para cumprir a Agenda 2030 foram prejudicados pela Pandemia, destacando os ODSs 1,2, 3, 4 e 8 em bloco, ODS 10 e os ODSs $13,14,15$ e 17.

O ODS 1 - Erradicação da Pobreza; 2 - Fome zero e agricultura sustentável; 3 - Saúde e bem estar; 4 - Educação de qualidade; 8 - Trabalho decente e crescimento econômico foram atingidos em cheio pela pandemia. Aumento da pobreza e da fome nos países em desenvolvimento com piora da saúde em todos os níveis e da educação devido a necessidade do ensino remoto, excluindo grande parte da população que não possui computador, tablet ou celular nem acesso à internet com a mínima qualidade. A pandemia causou fechamento de vários postos de serviço deixando uma legião de desempregados e recessonas economias de todos os países.

O ODS 10 - Redução das desigualdades ficou escancarado nos países em desenvolviment o onde as classes do topo da pirâmide puderam se isolar socialmente, acessar hospitais de qualidade, apesar de não ser suficiente quanto as perdas de ente queridos. Já as classes de menor poder aquisitivo viram seus entes queridos adoecerem e morrerem as vezes sem assistência alguma. Suas crianças estão fora da escola e a fome bate à porta da maioria. Esse objetivo está seriamente ameaçado dentro da política do salve-se quem puder. A situaçãoé preocupante e de difícil solução.

O ODS 13 - Ação climática que compreende diretamente as mudanças climáticas: inicialmente no mundo todo houve uma redução no aumento contínuo dos GEE, devido à paralisação das atividades econômicas não essenciais como algumas indústrias e transportes (WMO, 2020). Em 


\title{
Revista Científica ANAP Brasil
}

\author{
ISSN 1984-3240 - Volume 14, número 32, 2021
}

contrapartida, a crise econômica causada pela Pandemia pode desencadear a necessidade de acelerar a produção industrial e adicionar quantidades incríveis de GEE à atmosfera, colocando em risco os compromissos no âmbito do Acordo de Paris.

Similarmente, o ODSs 14 e 15 - Vida na Agua e Vida na Terra, respectivamente, devido ao agravamento da falta de recursos para aplicar em áreas protegidas, atingiu unidades de conservação tanto na agua como na terra. Houve incremento de microplásticos na água e no solo. Exemplificativamente, no Brasil, devido à fragilidade da agenda ambiental e enfraquecimento dos órgãos fiscalizadores, agravaram-se as queimadas e desmatamentos no Cerrado e na Amazônia com grandes perdas de habitat e de animais que não conseguiram se esquivar nas grandes queimadas. Foi um desastre ambiental nunca visto com participação da sociedade civil na tentativa de mitigar os efeitos.

Finalmente o ODS 17: Parcerias para atingir o objetivo, teve impactos na eficiência dos acordos internacionais, surgindo a necessidade de reconsiderar as parcerias regionais e globais (Cheval et al., 2020). Um caso contundente foi quanto as questões do acesso as vacinas contra a COVID-19 com um capítulo envolvendo a enorme dependência do Brasil com relação a Índia e a China. Vários países anteviram esse problema e se adiantaram como Israel, EUA e Chile. Suas economias poderão se recuperar mais rapidamente poupando sofrimento as populações.

No entanto, alguns países propõem uma recuperação econômica com enfoque ambiental. Um exemplo são as iniciativas de mais de 200 governos locais no Japão. Na reunião de lançamento do movimento de ação climática "TIME TO ACT" (2021) eles reconheceram a ameaça da crise climática e propuseram ações para superar a crise causada pela COVID-19, sendo Tóquio o centro financeiro para promover o mercado global de finanças verdes.

Enquanto o Ministro do Meio Ambiente do Peru, Gabriel Quijandría, durante o lançamento do Processo de Atualização da Estratégia Nacional de Mudanças Climáticas até 2050, indicou que a pandemia da COVID-19 afeta indiretamente as mudanças climáticas por meio da recessão, destacando que a descarbonização requer um investimento adicional de aproximadamente US\$ 99 bilhões até 2050, menor do que o benefício econômico líquido de US\$ 161 bilhões até 2050.

\subsection{Os obstáculos}

O combate à mineração ilegal no Peru aparenta retardar o status de "informal" (extração de ouro com grande dano ambiental e comercialização livre), além de fragilidade na gestão de eventos climáticos como El Niño Costeiro e outras previsíveis emergências relacionadas às mudanças climáticas, como as geadas nas altas regiões andinas e as "temporadas" de incêndios florestais nas montanhas e floresta. O Governo do Peru e o Município Metropolitano de Lima nos últimos quatro anos têm desempenhado um papel antagônico no âmbito do NDC, priorizando obras de infraestrutura rodoviária de má qualidade, que têm agravado o problema da frota automotiva em Lima. Esquecendo assim a ordem do transporte terrestre, a promoção dos veículos não motorizados e o aumento das áreas verdes (Ráez, 2019).

O problema é refletido no World Air Quality Report (2019 World Air Quality Report PM 2.5 Ranking), que coloca ao Peru no primeiro lugar entre os países e Lima em segundo lugar entre as capitais da América Latina e do Caribe com pior qualidade do ar. O Peru registrou 23,28 $\mu \mathrm{g} / \mathrm{m}^{3}$ e Lima 23,7 $\mathrm{\mu g} / \mathrm{m}^{3}$ de $\mathrm{PM}_{2,5}$, considerando contaminação "moderada" o suficiente para causar problemas na saúde da população, principalmente aos mais vulneráveis aos produtos químicos ou poluentes e com problemas respiratórios pré-existentes. Porém, durante a 
pandemia o registro dos índices de qualidade do ar no país foi considerado "bom", já que Lima registrava concentrações de até $6,6 \mu \mathrm{g} / \mathrm{m}^{3}$ (+ IQAir, 2020).

Em relação a necessidade de encontrar soluções para o problema de transporte terrestre em Lima, houve a pressa de iniciar o projeto da linha 2 do trem elétrico de Lima. No entanto, existiram oposições à convocatória internacional para um projeto que não tinha um estudo de viabilidade, nem alternativas tecnológicas com suporte técnico-económico. Do mesmo modo, a formulação do projeto carecia de coerência com a realidade, como a chegada ao país dos primeiros trens importados da Itália em setembro de 2016, os quais ficarão armazenados por cerca de 6 anos até a sua utilização (Velásquez, 2018).

O cenário que vivemos hoje devido a COVID-19 tem gerado preocupação no mundo devido a rejeição ao transporte público, optando pelos veículos automotores particulares. Um estudo em Hubei, província da China em Wuhan, mostrou que esta doença se espalhou de uma pessoa para nove em uma única viagem de ônibus (Null and Smith, 2020).

\section{CONCLUSÕES}

A revisão das ações mais importantes que enquadram as Contribuições Nacionalmente Determinadas do Peru e as ações no contexto da pandemia da COVID-19 permitiu entender a importância da sustentabilidade nos planos de recuperação e reativação pós-pandemia.

O Peru ampliou suas metas em termos de mitigação de GEE, passando de $30 \%$ para $40 \%$ de sua redução total até 2030; em termos de adaptação das mudanças climáticas, procuraram construir um modelo baseado na gestão de riscos diante desses efeitos para reduzir a vulnerabilidade do país. Além disso, a articulação multissetorial permite manter um esquema participativo, político e técnico na gestão climática.

Durante a pandemia, várias atividades foram suspensas ou mais restritas; porém, a criação da Comissão de Alto Nível sobre Mudanças Climáticas, a Comissão Nacional sobre Mudanças Climáticas, a Plataforma dos Povos Indígena e a plataforma digital Cadastro Nacional de Medidas de Mitigação (RENAMI) criada durante a pandemia, tem como objetivo dar continuidade ao compromisso implementado, monitorando, financiando e atualizando as NDC. As ferramentas como inventários nacionais de GEE, documentos que comunicam a renovação cíclica das NDC, se relatadas oportunamente, fortalecerão o cumprimento do compromisso do Peru com as mudanças climáticas. Isto inclui a melhoria do controle da qualidade do ar, que no ano 2019 refletiu possíveis consequências para a saúde da população, e também sendo mais vulneráveis diante a pandemia da COVID-19.

A troca de experiências entre os países comprometidos com o Acordo de Paris melhora a gestão, a política e as medidas que serão atualizadas nas NDC fortalecendo seus compromissos.

Atenuar os impactos da pandemia sobre a Agenda 2030 e seus 17 ODSs será um desafio maior para toda a humanidade visto o aumento da pobreza, da fome, redução da saúde e do acesso à educação de qualidade, o desemprego crescente e o recesso econômico que escancarou as desigualdades principalmente nos países em desenvolvimento.

A situação atual da pandemia nos leva a meditar sobre o consumo excessivo de certos recursos, as atitudes que podemos adotar para melhorar a qualidade do meio ambiente para que junto com o sustento científico possa aperfeiçoar a gestão das mudanças climáticas, 
considerando a descarbonização, porque não é apenas a economia de um país que está em jogo, mas a sobrevivência digna da humanidade.

\section{REFERÊNCIAS}

CAMMELLI, F.; ANGELSEN, A. Amazonian farmers' response to fire policies and climate change. Ecological Economics, v. 165, p. 106359, 2019.

CHEVAL, S. et al. Observed and Potential Impacts of the COVID-19 Pandemic on the Environment. International Journal of Environmental Research and Public Health, v. 17, n. 11, p. 4140, 2020.

DOUROJEANNI, M. [Opinión] Impactos ambientales de la pandemia en el Perú. Disponível em: <https://www.actualidadambiental.pe/opinion-impactos-ambientales-de-la-pandemia-en-el-peru/>. Acesso em: 8 jan. 2021.

EUROCLIMA+ promueve diálogo entre países latinoamericanos para fortalecer la acción climática en la región. Disponível em: <http://euroclimaplus.org/noticias-eventos-gobernanza/noticias-gobernanza/162-euroclimapromueve-dialogo-entre-paises-latinoamericanos>. Acesso em: 8 jan. 2021.

GOBIERNO DEL PERÚ. Informe Final del Grupo de Trabajo Multisectorial de naturaleza temporal encargado de generar información técnica para orientar la implementación de las Contribuciones Nacionalmente Determinadas (GTMNDC). Lima, 2018.

GOBIERNO DEL PERÚ. Contribuciones Determinadas a nivel nacional del Perú. Lima, 2020.

JARA, A. Es necesario revisar el proyecto de la línea 2 del tren eléctrico de Lima. Disponível em: $<$ https://revistas.urp.edu.pe/index.php/Perfiles_Ingenieria/article/view/2368>. Acesso em: 11 mar. 2021.

Peru. Decreto Supremo № 006-2020-MINAM, de 4 de julio de 2020. Decreto Supremo que crea la Comisión Multisectorial de carácter permanente denominada "Comisión de Alto Nivel de Cambio Climático (CANCC)". Diário Oficial El Peruano: p. 7-9.

Peru. Decreto Supremo № 013-2019-MINAM, de 31 de dezembro de 2019. Decreto Supremo que aprueba el Reglamento de la Ley № 30754, Ley Marco sobre Cambio Climático. Diário Oficial El Peruano: p. 2-21.

Peru. Ley № 30754, de 18 de abril de 2018. Ley Marco sobre Cambio Climático. Diário Oficial El Peruano: p. 3-9.

Lima Air Quality Index (AQI) and Peru Air Pollution | AirVisual. Disponível em: <https://www.iqair.com/peru/lima>. Acesso em: 10 mar. 2021.

LIU, Z. et al. Near-real-time monitoring of global $\mathrm{CO} 2$ emissions reveals the effects of the COVID-19 pandemic. Nature Communications, v. 11, n. 1, 2020.

Manuel Pulgar Vidal. El acuerdo de parís: El largo proceso hacia el éxito. Lima: Ministerio del Ambiente, 2016.

MINISTERIO DEL AMBIENTE. La Contribución Nacional del Perú - iNDC: agenda para un desarrollo climáticamente responsable. Lima: Ministerio del Ambiente, 2016.

MINISTERIO DEL AMBIENTE. Inventario Nacional de Gases de Efecto Invernadero del año 2014. Lima, 2019.

Naciones Unidas. Convención Marco de las Naciones Unidas sobre el Cambio Climático. [s.I:s.n.].

NULL, S.; SMITH, H. COVID-19 Could Affect Cities for Years. Here Are 4 Ways they're Coping Now. I. Disponível em: <https://thecityfix.com/blog/covid-19-affect-cities-years-4-ways-theyre-coping-now-schuyler-null-hillary-smith/>. Acesso em: 10 ago. 2020.

Ráez, E. Cambio climático en el Perú: contribuciones nacionales, su definición y estado de avance. Gamagráfica S.A, 2019.

República Del Perú. Contribución Prevista y Determinada a Nivel Nacional (iNDC). Lima, 2015. 
Revista Científica ANAP Brasil

ISSN 1984-3240 - Volume 14, número 32, 2021

VÁSQUEZ BACA, U.; GAMIO AITA, P. Transición energética con energías renovables para la seguridad energética en el Perú: una propuesta de política pública resiliente al clima. Espacio y Desarrollo, n. 31, p. 195-224, 2018. 